\title{
In Vitro Skin Permeation of Morphine Hydrochloride during the Finite Application of Penetration-Enhancing System Containing Water, Ethanol and $l$-Menthol
}

\author{
Yasunori Morimoto, ${ }^{*, a, b}$ Yoshio WadA, ${ }^{b}$ Toshinobu SeKI, ${ }^{a, b}$ and Kenji SugibaYashi ${ }^{a, b}$ \\ Faculty of Pharmaceutical Sciences, Josai University, ${ }^{a}$ and Research Institute of TTS Technology, ${ }^{b}$ 1-1 Keyakidai, Sakado, \\ Saitama 350-0295, Japan. Received August 6, 2001; accepted October 2, 2001
}

\begin{abstract}
The effects of composition of applied solutions, containing water, ethanol (EtOH) and $l$-menthol (LM) as penetration enhancers, on the in vitro permeation of morphine hydrochloride (MPH) through excised hairless rat skin were examined in finite application experiments. Three of the five different applied solutions contained almost saturated LM and two contained levels of LM below the limit of solubility. Despite similar pseudo steadystate fluxes (maximum fluxes observed) of MPH from the solutions, lag time for the permeation of MPH from the saturated systems was shorter than that from the unsaturated systems. Lag times for the permeation of EtOH and $L M$ from the saturated systems were also shorter than those from the unsaturated systems. Thermodynamic activity of LM is important for the enhancing effect against MPH permeation. At the beginning for the permeation experiment, the activity of LM in the unsaturated systems was lower than that in the saturated solutions. As the skin permeability of EtOH was higher than that of other components, the content of EtOH in the applied solution gradually decreased with time, while the activity of LM increased eventually showing a sufficient enhancing effect. Solvent drag effect was not important for the permeation of MPH, since penetration rate of MPH was independent of the time course of that of EtOH. The amount of LM migrating into skin appeared to be the most important parameter for the penetration-enhancing effect of the mixed system in the in vitro permeation of MPH through excised hairless rat skin.
\end{abstract}

Key words ethanol; skin penetration enhancer; finite application; morphine hydrochloride; $l$-menthol

We previously discussed the possibility of transdermal application of morphine hydrochloride (MPH) ${ }^{1-3)}$ and examined a mixed solvent system containing water, ethanol (EtOH) and $l$-menthol (LM) as a skin penetration-enhancing system. ${ }^{4}{ }^{-8)}$ The phase condition of the system markedly affected the penetration enhancing effect. ${ }^{6}$ The saturated LM in the applied aqueous solvent system was necessary to obtain a strong enhancing effect, but an excessive amount of LM, which made an o/w emulsion, decreased the enhancing effect. When a finite amount of the system was applied to skin, the system composition on skin changed over time due to the high skin permeability of EtOH. ${ }^{9,10)}$ The composition change of the system must be considered to design a useful transdermal delivery system containing water, EtOH and LM as penetration enhancers.

In this study, a finite amount of the mixed solvent system containing MPH, water, EtOH and LM were applied to excised hairless rat skin, and MPH, EtOH and LM permeation was examined. 1,3-Butylene glycol (BG) was used as one more component to control hydrophilicity or lipophilicity of the mixed system. ${ }^{11)}$ Five different formulas were used for the application systems (Table 1). Three contained almost saturated LM, and the initial content of LM in the rest was below the limit of solubility of LM. Polyethyleneoxide E-45 (PEO) was used as a neutral polymer to increase viscosity of the systems.

\section{MATERIALS AND METHODS}

Materials MPH and naloxone hydrochloride were purchased from Takeda Pharmaceutical Co. (Osaka, Japan) and Sigma Chemical Industries (St. Louis, MO, U.S.A.), respectively. LM was purchased from Wako Pure Chemical Industries (Osaka). All other chemicals were of reagent grade and were used without further purification.

In Vitro Skin Permeation Experiments The abdominal skin of male WBN/ILA-Ht hairless rats $(150-200 \mathrm{~g}$, Ishikawa Laboratory Animals, Saitama, Japan) was excised and mounted in a vertical diffusion cell with a receiver volume of $2.8 \mathrm{ml}$ and an effective diffusion area of $0.95 \mathrm{~cm}^{2}$. The receiver cell was filled with distilled water and the cell set was kept at $37^{\circ} \mathrm{C}$. Two hundred milligrams of each solution shown in Table 1 was applied to the skin. One milliliter of receiver solution was withdrawn at predetermined times for $24 \mathrm{~h}$ to determine concentrations of MPH, LM and EtOH. The same volume of water was added to the receiver compartment to keep the volume constant.

Analysis The concentration of MPH was assayed by an HPLC system (pump, LC-9A; UV detector, SPD-6A; Shimadzu Seisakusho, Kyoto, Japan). The column used was a LiChrospher 100 RP-18(e) $5 \mu \mathrm{m} \quad(4.0 \times 250 \mathrm{~mm}$, Kanto Chemical Co., Tokyo, Japan). A mixture of $0.1 \%$ phosphoric acid-acetonitrile $(65: 35)$ containing $5 \mathrm{~mm}$ sodium dodecylsulfate was used as the mobile phase, and elution was performed at $40{ }^{\circ} \mathrm{C}$ with a flow rate of $1.2 \mathrm{ml} / \mathrm{min}$. Aliquots of $200 \mu \mathrm{l}$ of the receiver solution were withdrawn and mixed with the same volume of methanol containing naloxone hydrochloride as an internal standard, and then the mixture was centrifuged at $5000 \mathrm{~g}$ for $10 \mathrm{~min}$. Aliquots of the supernatant (20 $\mu \mathrm{l})$ were injected into the HPLC system. The UV detector was operated at $230 \mathrm{~nm}$.

Gas chromatography (GC) with a flame ionization detector (GC-6A, Shimadzu Seisakusho) was used for determination of LM and EtOH. OV-17 was used as the column and GC was operated at a column temperature of $130^{\circ} \mathrm{C}$, and injector and detector temperature of $160^{\circ} \mathrm{C}$ for $\mathrm{LM}$ analysis. On the other hand, Gasukuropak 54 was used as the column and GC was operated at a column temperature of $140^{\circ} \mathrm{C}$, and injector 
and detector temperature of $180^{\circ} \mathrm{C}$ for $\mathrm{EtOH}$ analysis. Aliquots of $200 \mu \mathrm{l}$ of the receiver solution were mixed with the same volume of dioxane containing $n$-propanol and $l$-carvone as internal standards for EtOH and LM, respectively, and then the mixture was centrifuged at $5000 \mathrm{~g}$ for $10 \mathrm{~min}$. Aliquots of the supernatant $(1 \mu \mathrm{l})$ were injected under each condition for EtOH and LM.

\section{RESULTS AND DISCUSSION}

Five different solutions shown in Table 1 were used for application of MPH. As LM is an essential oil, it is insoluble in water. EtOH and BG act as solubilizers for LM. Since BG is a little more hydrophilic than $\mathrm{EtOH}$ (the solubility parameter: $\left.\mathrm{BG}=28.15\left(\mathrm{~J} / \mathrm{cm}^{3}\right)^{1 / 2}, \mathrm{EtOH}=26.14\left(\mathrm{~J} / \mathrm{cm}^{3}\right)^{1 / 2}\right)$, the replacement of EtOH with BG makes the system more hydrophilic. ${ }^{11)}$ However, the difference between EtOH and BG in the solubilizing effect for LM was small. Five percents of LM in the systems was solubilized when total content of $\mathrm{EtOH}$ and $\mathrm{BG}$ was $50 \%$. Therefore, three contained almost saturated LM, and the initial content of LM in the rest was below the limit of solubility of LM in the system.

Two hundred milligrams of each solution was applied to the excised hairless rat skin and MPH, EtOH and LM skin permeations were examined. The time course of permeation flux of MPH is shown in Fig. 1. In the cases of application of the LM-saturated systems, the maximum values of the flux were observed within $4 \mathrm{~h}$ and the pseudo steady-state fluxes gradually decreased thereafter. Although the EtOH/BG ratio was different in the LM-saturated systems, MPH permeation profiles from these systems were similar, suggesting a similarity of $\mathrm{EtOH}$ and $\mathrm{BG}$ effect on the skin. On the other hand, a lag time of about $2 \mathrm{~h}$ for the permeation of MPH was observed following the application of LM-solubilized systems. After the lag time, the flux of MPH increased rapidly. Although the permeation profiles of MPH in the initial phase were different, the maximum values of the flux and the permeation profiles in the following phase of MPH were similar with application of all systems.

Our previous study showed that saturated LM in the applied system is necessary to obtain a strong enhancing effect. ${ }^{6}$ In finite applications, the solubility of LM changed due to the escape of permeable components such as EtOH from the applied solutions into the skin, and thereby the activity and penetration-enhancing effects of LM changed over passing time. In addition, if a solvent drag effect of EtOH contributed to enhancement of MPH permeation, the time course of changes in the permeation rate of MPH would depend on that of EtOH. ${ }^{12,13)}$ The delayed enhancing effect with application of LM-solubilized systems suggested that the activity of LM and its changes in the applied solutions are important for the enhancing effect of the system. The permeation of EtOH and LM through the skin was also determined to understand the mode of action of the penetration enhancing systems. The profiles of the permeation rate of $\mathrm{EtOH}$ and LM are shown in Figs. 2 and 3, respectively. The permeation rates of EtOH were higher than those of MPH and LM. Comparison of the initial high permeation rates of EtOH from the LM-saturated systems with that from the unsaturated systems suggested a marked contribution of solvent drag of EtOH. Although the maximum flux of $\mathrm{EtOH}$ permeation from the
Table 1. Composition of the Solution for Application to the Skin

\begin{tabular}{ccccccc}
\hline \hline \multirow{2}{*}{ Rp. \# } & \multicolumn{5}{c}{ Content (\%) } & \multirow{2}{*}{ PC $^{a)}$} \\
\cline { 2 - 5 } & EtOH & BG & LM & MPH & PEO & \\
\hline 1 & 10 & 40 & 5 & 1 & 3 & $\mathrm{~S}^{b)}$ \\
2 & 20 & 30 & 5 & 1 & 3 & $\mathrm{~S}^{c)}$ \\
3 & 20 & 40 & 5 & 1 & 3 & $\mathrm{U}^{c)}$ \\
4 & 30 & 20 & 5 & 1 & 3 & $\mathrm{~S}$ \\
5 & 30 & 30 & 5 & 1 & 3 & $\mathrm{U}$ \\
\hline
\end{tabular}

Water was added to $100 \%$. a) Phase condition, $b$ ) LM-saturated, $c$ ) LM-unsaturated.

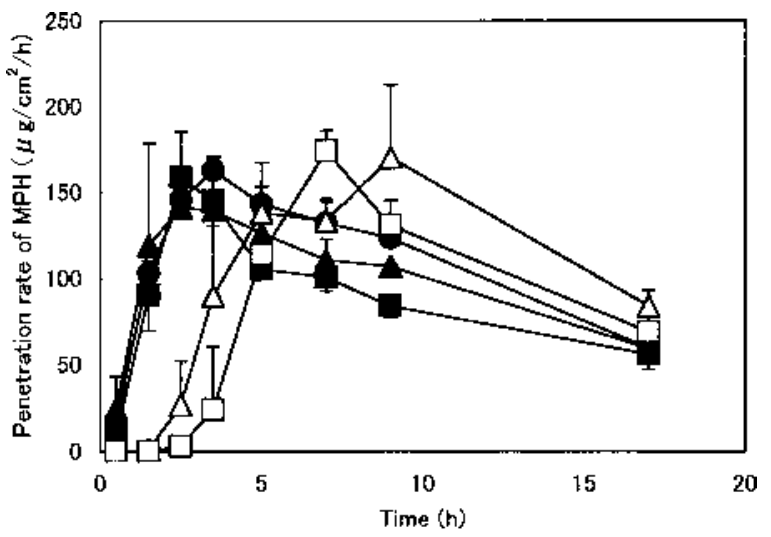

Fig. 1. Time Course of Changes in the Permeation Rate of MPH through Excised Hairless Rat Skin

๑; EtOH $10 \%$, BG $40 \%$, LM 5\% (Rp. \# 1 in Table 1): $\mathbf{\Delta}$; EtOH $20 \%$, BG 30\%, LM $5 \%$ (Rp. \# 2 in Table 1): $\triangle$; EtOH $20 \%$, BG $40 \%$, LM $5 \%$ (Rp. \# 3 in Table 1): $\mathbf{\square}$; EtOH $30 \%$, BG 20\%, LM 5\% (Rp. \# 4 in Table 1): $\square$; EtOH $30 \%$, BG 30\%, LM 5\% (Rp. \# 5 in Table 1). Points represent the means \pm S.D. $(n=3)$.

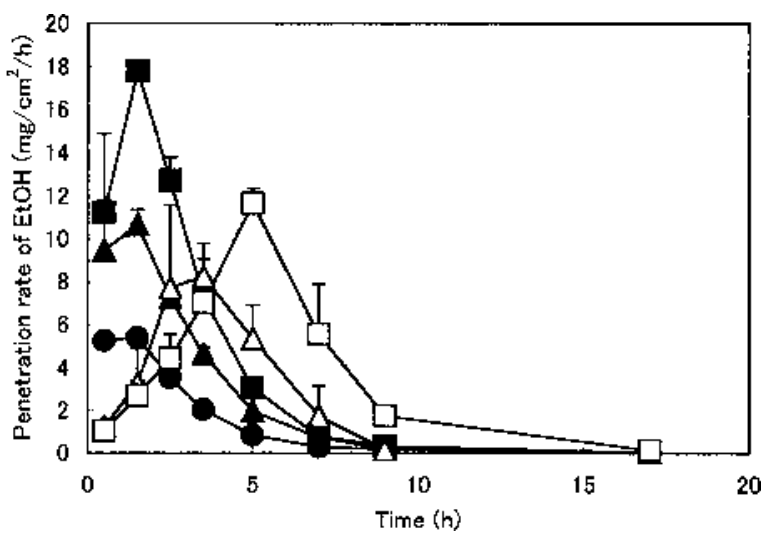

Fig. 2. Time Course of Changes in the Permeation Rate of EtOH through Excised Hairless Rat Skin

- EtOH $10 \%$, BG 40\%, LM 5\% (Rp. \# 1 in Table 1): $\mathbf{\Delta}$; EtOH 20\%, BG 30\%, LM $5 \%$ (Rp. \# 2 in Table 1): $\triangle$; EtOH $20 \%$, BG 40\%, LM 5\% (Rp. \# 3 in Table 1): EtOH $30 \%$, BG 20\%, LM 5\% (Rp. \# 4 in Table 1): $\square$; EtOH $30 \%$, BG $30 \%$, LM $5 \%$ (Rp. \# 5 in Table 1). Points represent the means \pm S.D. $(n=3)$.

LM-saturated systems was proportional to initial content of $\mathrm{EtOH}$ in the systems and most of the EtOH applied had permeated through the skin within $10 \mathrm{~h}$, however, the maximum flux of MPH was independent of the initial content of EtOH and the permeation of MPH continued beyond $10 \mathrm{~h}$ after the application. Therefore, the contribution of the solvent drag effect of EtOH to MPH permeation could be small in these cases. In addition, since the initial EtOH permeation from the LM-saturated systems was proportional to EtOH content and 


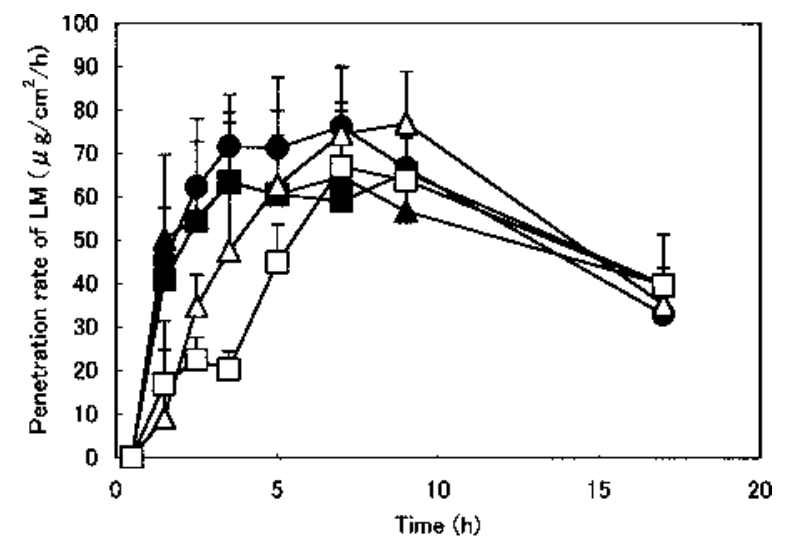

Fig. 3. Time Course of Changes in the Permeation Rate of LM through Excised Hairless Rat Skin

-; EtOH $10 \%$, BG $40 \%$, LM 5\% (Rp. \# 1 in Table 1): $\mathbf{\Delta}$; EtOH $20 \%$, BG 30\%, LM $5 \%$ (Rp. \# 2 in Table 1): $\triangle$; EtOH $20 \%$, BG $40 \%$, LM $5 \%$ (Rp. \# 3 in Table 1): EtOH $30 \%$, BG 20\%, LM 5\% (Rp. \# 4 in Table 1): $\square$; EtOH $30 \%$, BG $30 \%$, LM $5 \%$ (Rp. \# 5 in Table 1). Points represent the means \pm S.D. $(n=3)$.

was independent of BG content, the effect of BG on the permeation of EtOH may be small. The permeation of LM through the skin from the LM-saturated systems was higher than that from the LM-solubilized systems in the initial phase and the permeation of LM in the later phase from the all systems was similar. LM changes barrier function in the stratum corneum. ${ }^{14,15)}$ The continuous permeation of MPH in the later phase could be related to that of LM. Amount of LM migrated into the skin appeared to be the most important parameter for the penetration-enhancing effect of the mixed system in MPH permeation through excised hairless rat skin.

Since this study is incomplete and partial, complete mechanism on the penetration-enhancing effect of each component and the interaction between the components have not been understood yet. As demonstrated in this study, however, changes in the composition of the applied solution should be considered in the cases of finite application of enhancing systems containing EtOH and LM. In addition, the amount of solution applied to skin should be related not only to bioavil- ability $^{16,17)}$ of the drug applied but also to skin irritancy of EtOH in the system. ${ }^{18)}$ The composition and volume of drugreservoir solution should be optimized to design a useful transdermal delivery system containing water, EtOH and LM as penetration enhancers. For the optimization, additional studies of enhancing mechanism in detail should be needed.

\section{REFERENCES}

1) Hosoya O., Sano M., Wada Y., Seki T., Sugibayashi K., Juni K., Morimoto Y., Chem. Pharm. Bull., 46, 882-885 (1998).

2) Matsuzawa T., Wada Y., Shimoyama M., Nakajima K., Seki T., Sugibayashi K., Morimoto Y., Biopharm. Drug Dispo., 15, 665-678 (1994).

3) Sugibayashi K., Sakanoue C., Morimoto Y., Selective Cancer Ther., 5, 119-128 (1989).

4) Sugibayashi K., Kobayashi D., Nakagaki E., Hatanaka T., Inoue N., Kusumi S., Kobayashi M., Kimura M., Morimoto Y., Int. J. Pharmaceut., 113, 189-197(1995).

5) Kobayashi D., Matsuzawa T., Sugibayashi K., Morimoto Y., Kimura M., Pharm. Res., 11, 96-103 (1994).

6) Wada Y., Nakajima K., Inada H., Sugibayashi K., Morimoto Y., J. Pharm. Sci. Technol. Jpn., 54, 1-9 (1994).

7) Morimoto Y., Sugibayashi K., Kobayashi D., Shouji H., Yamazaki J., Kimura M., Int. J. Pharmaceut., 91, 9-14 (1993).

8) Wada Y., Nakajima K., Yamazaki J., Seki T., Sugibayashi K., Morimoto Y., Biol. Pharm. Bull., 16, 600-603 (1993).

9) Chen S. X., Lostritto R. T., J. Pharm. Sci., 86, 739-746 (1997).

10) Hori M., Maibach H. I., Guy R. H., J. Pharm. Sci., 81, 330-333 (1992).

11) Sano M., Hosoya O., Taoka S., Seki T., Kawaguchi T., Sugibayashi K., Juni K., Morimoto Y., Chem. Pharm. Bull., 47, 1044-1046 (1999).

12) Comfort A. R., Shevchuk I., Otte J. H., Dinh S. M., J. Control. Release, 34, 193-201 (1995).

13) Friend D. R., Smedley S. I., Int. J. Pharmaceut., 97, 39-46 (1993).

14) Maitani Y., Shimada K., Nagai T., Chem. Pharm. Bull., 44, 403-408 (1996).

15) Obata Y., Takayama K., Maitani Y., Machida Y., Nagai T., Biol. Pharm. Bull., 16, 312-314 (1993).

16) Jin Y., Seki T., Morimoto Y., Juni K., Drug Dev. Ind. Pharm., 26, 193-198 (2000).

17) Tata S., Flynn G. L., Weiner N. D., J. Pharm. Sci., 84, 688-691 (1995).

18) Seki T., Wada Y., Kobayashi N., Juni K., Morimoto Y., Drug Delivery System, 15, 121-126 (2000). 